\title{
Plasma concentrations of D-dimer and outcome of in vitro fertilization
}

\author{
Marcello Di Nisio ${ }^{1,2}$, Ettore Porreca ${ }^{3}$, Valeria Di Donato ${ }^{3}$ and Gian Mario Tiboni ${ }^{3 *}$
}

\begin{abstract}
Background: The activation of blood coagulation could contribute to the failure of in-vitro fertilization (IVF) techniques. The aim of this study was to assess the predictive value of D-dimer levels for pregnancy outcome in women undergoing IVF.

Findings: A prospective study was performed in 105 women undergoing IVF. D-dimer was measured before and one week after the administration of recombinant human chorionic gonadotropin (r-hCG). The primary outcome of the study was clinical pregnancy. The mean age was 36 years (range 26 to 43 years). The main indications for IVF were infertility due to a tubaric $(n=21,20 \%)$ or male factor $(n=37,35 \%)$ and idiopathic infertility ( $n=30,29 \%$ ) which altogether accounted for $84 \%$ of the total. Clinical pregnancy was achieved by 40/105 (38\%) women of whom 32 (80\%) delivered a live child. On the day of $r-h C G$ administration, D-dimer concentrations were significantly higher in patients not achieving a clinical pregnancy $(141 \mathrm{ng} / \mathrm{dL}$ vs. $115 \mathrm{ng} / \mathrm{dL}, \mathrm{p}=0.035)$ which remained statistically significant after correction for age and indications for IVF in multivariable analysis $(p=0.032)$. One week after $r-h C G$, the levels of D-dimer were significantly increased both in women with and without a clinical pregnancy with no differences between the groups $(748 \mathrm{ng} / \mathrm{dL}$ vs. $767 \mathrm{ng} / \mathrm{dL}, \mathrm{p}=0.88)$.

Conclusions: D-dimer concentrations seem to predict a higher risk of pregnancy failure in women undergoing IVF. If confirmed in future prospective studies, D-dimer could help identifying a group of patients who could benefit from prophylaxis to increase the pregnancy success rate.
\end{abstract}

Keywords: In-vitro fertilization, Clinical pregnancy, D-dimer, Hypercoagulability

\section{Background}

The average pregnancy rate after in-vitro fertilization (IVF) remains as low as 30\% [1]. One of the possible mechanisms behind the high failure rate is the unsuccessful implantation or placentation due to hypercoagulability causing thrombosis of maternal vessels with reduced perfusion of the intervillous space and placentation failure [2]. A number of studies evaluated the causal relationship between states of hypercoagulability and outcomes of IVF reporting conflicting findings, as summarised in a recent systematic review of the literature [3].

The hormonal milieu resulting from the use of contraceptive pills or hormone replacement therapy has been clearly associated with hypercoagulability $[4,5]$. By contrast,

\footnotetext{
*Correspondence: tiboni@unich.it

${ }^{3}$ Department of Medicine and Aging, University "G. D'Annunzio" of Chieti-Pescara, Chieti, Italy

Full list of author information is available at the end of the article
}

only few studies examined the impact on haemostatic parameters of supra-physiological oestrogen levels as seen during IVF [6]. Although the interpretation of the data remains difficult due to the relatively small size of the studies and the heterogeneity of IVF protocols, the available evidence suggests that ovarian stimulation during IVF is associated with increased concentrations of coagulation factors and impairment of endogenous anticoagulants. All these haemostatic changes seem amplified in cases of excessive ovarian response as it occurs in the ovarian hyperstimulation syndrome (OHSS) [7]. Interestingly, preliminary observations suggested that haemostatic markers such as D-dimer are associated with an unfavourable pregnancy outcome in women with OHSS [7].

The aim of this prospective study was to evaluate the association between plasma D-dimer levels and IVF outcome.

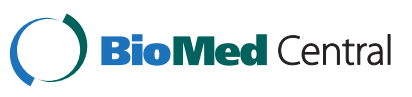




\section{Methods \\ Patients}

Women attending the Assisted Reproduction Unit of the Ortona General Hospital and undergoing IVF from January 2011 to December 2012 were eligible for the study. Indications for IVF treatment included anovulation, endometriosis, tubal factor, male factor, mixed factor and unexplained infertility. Exclusion criteria were the ongoing use of anticoagulants at prophylactic or therapeutic doses or unwillingness to provide consent for the participation in the study. High responders that were coasted to prevent OHSS were also excluded. The study was conducted in compliance with the Helsinki Declaration and informed consent was obtained from all participants.

\section{Ovarian stimulation protocol}

Gonadotropin Releasing Hormone (GnRH) agonist or antagonist were used to prevent premature luteinizing hormone (LH) surges. Ovarian stimulation was performed using daily subcutaneous injections of recombinant folliclestimulating hormone (r-FSH; Puregon, Merk Sharpe and Dome, Germany; Gonal F, Merk Serono, Switzerland) at doses ranging from $75 \mathrm{IU}$ to $450 \mathrm{IU}$ depending on the woman's age, the antral follicle count (AFC), and the basal (day 3) FSH circulating level. Controlled ovarian stimulation was started on day 3 of full menstrual flow if pituitary suppression was complete, as shown by absence of ovarian cysts, serum estradiol $<50 \mathrm{pg} / \mathrm{ml}$ and endometrial thickness $<3 \mathrm{~mm}$. Gonadotropin dosage was adjusted according to the individual response. Recombinant human chorionic gonadotropin ( $\mathrm{r}$-hCG; Ovitrelle, Merk-Serono, Switzerland ) was administered when at least three leading follicles reached $18 \mathrm{~mm}$. Oocyte pick-up was scheduled 36 hours after r-hCG injection. All the collected oocytes were denuded and their nuclear maturation stage determined. All the in-vitro fertilizations were performed by intracytoplasmic sperm injection (ICSI). Embryo transfer was performed 72-76 hours after ovum pick-up. A luteal phase support of $600 \mathrm{mg} /$ day micronized intravaginal progesterone (Progefik, Effikitalia, Italy) was started the day after oocyte retrieval and maintained until a pregnancy test was performed, or until the seventh week's gestation if the patient tested positive. Pregnancy test was performed by dosing serum $\beta$ hCG levels, 14 days after embryo transfer. Clinical pregnancy was defined as the presence of a gestational sac with accompanying fetal heart beat as observed by ultrasound at seven weeks gestation.

\section{Blood collection}

Venous blood was collected from an antecubital vein without venous stasis using a 19-gauge butterfly needle. Blood withdrawal was planned 48 hours before the administration of r-hCG (maximal estrogens concentration) and between eight to ten days afterwards. Blood samples for D-dimer test were collected into tubes containing a mixture of sodium citrate, centrifuged for 15 minutes at $3,000 \times \mathrm{g}$, and stored at $-80^{\circ} \mathrm{C}$ until further analysis. $\mathrm{D}$-dimer concentrations were measured by a latex quantitative assay (D-dimer Plus assay, Dade Behring, Marburg, Germany) with a cut-off value of $200 \mathrm{ng} / \mathrm{mL}$ for an abnormal test result according to the manufacturer indications.

\section{Statistical analysis}

Information on baseline characteristics, risk factors, and treatments were analyzed by means of descriptive statistics. Variables with a non-parametric distribution were presented as medians (range) and those with a parametric distribution as means ( \pm standard deviation). Differences between women achieving a clinical pregnancy and women with a pregnancy failure were tested with the Mann-Whitney U test or the T-test, as appropriate. Variables with a statistical significance corresponding to a $\mathrm{p}<0.15$ in univariate analysis were assessed in multivariate logistic regression analysis. D-dimer levels were analysed as a continuous variable and according to the cut-off of the assay for positivity (200 ng/dL) comparing values above and below the cut-off. All statistical analyses were performed using SPSS software (version 20; SPSS Inc, Chicago, Illinois).

\section{Results}

The clinical characteristics of the study population are shown in Table 1 . The mean age was 36 years (26 to 43 years). In over two thirds of the cases infertility was idiopathic or the consequence of a male or tubal factor. Overall, $5(4.8 \%)$ of 105 women achieved a biochemical pregnancy, and $40(38 \%)$ obtained a clinical pregnancy of whom 32 (80\%) delivered a live child which was pre-term (before 37 weeks gestation) in 23 (72\%) (Table 2). Twin pregnancy occurred in 9/40 (22\%) women, and in one case pregnancy was complicated by intra uterine growth restriction. Seven women aborted before the 12th week gestation (17\%) and one experienced an extra uterine pregnancy. There were no thromboembolic complications during the study and pharmacological thromboprophylaxis was not administered to any patient during IVF.

Women achieving clinical pregnancy were similar for age, vascular risk factors, indication for IVF, and IVF procedures to women who had a pregnancy failure (Tables 1 and 2).

\section{D-dimer levels}

Circulating levels of D-dimer measured just before the administration of $\mathrm{r}$-hCG were significantly higher in women with a pregnancy failure $(141 \mathrm{ng} / \mathrm{mL}$, range 42 to $499 \mathrm{ng} / \mathrm{mL}$ ) compared to those achieving a clinical pregnancy $(115 \mathrm{ng} / \mathrm{mL}$, range 16 to $215 \mathrm{ng} / \mathrm{mL} ; \mathrm{p}=0.035)$ 
Table 1 Clinical and demographic characteristics of the study population

\begin{tabular}{|c|c|c|c|}
\hline \multirow[t]{2}{*}{ Characteristic } & \multicolumn{2}{|c|}{ Viable pregnancy } & \multirow[t]{2}{*}{$P$ value } \\
\hline & Yes $(n=40)$ & No $(n=65)$ & \\
\hline Age, years & $36(27-43)$ & $36(26-43)$ & 0.97 \\
\hline BMl, kg/m2 & $22(17-39)$ & $21(16-39)$ & 0.26 \\
\hline Smoking & $7(17)$ & $11(17)$ & 0.94 \\
\hline Arterial hypertension & 0 & $1(1)$ & 0.43 \\
\hline Diabetes & 0 & 0 & - \\
\hline $\begin{array}{l}\text { History of cardio-cerebrovascular } \\
\text { disease }\end{array}$ & $1(2)$ & 0 & 0.20 \\
\hline $\begin{array}{l}\text { History of venous } \\
\text { thromboembolism }\end{array}$ & 0 & 0 & - \\
\hline Indication for IVF & & & 0.26 \\
\hline Male factor & $11(27)$ & $26(40)$ & \\
\hline Tubaric & $8(20)$ & $13(20)$ & \\
\hline Idiopathic & $15(37)$ & $15(23)$ & \\
\hline Ovulatory & $5(12)$ & $4(6)$ & \\
\hline Endometriosis & $1(2)$ & $5(8)$ & \\
\hline Recurrent miscarriage & 0 & $2(3)$ & \\
\hline Previous pregnancy & $8(20)$ & $9(14)$ & 0.41 \\
\hline Previous miscarriage & & & 0.62 \\
\hline No & $37(92)$ & $57(88)$ & \\
\hline 1 & $1(2.5)$ & $5(8)$ & \\
\hline 2 & $1(2.5)$ & $2(3)$ & \\
\hline 3 & $1(2.5)$ & $1(1)$ & \\
\hline$\geq 3$ & 0 & 0 & \\
\hline
\end{tabular}

Number of previous cycles

\begin{tabular}{lccc}
0 & 32 & 30 & \\
1 & 8 & 20 & \\
2 & 3 & 3 & \\
3 & 4 & 3 & \\
$>3$ & 1 & 1 & \\
Embryos transferred & & & 0.98 \\
1 & $1(2)$ & $2(3)$ & \\
2 & $4(10)$ & $7(11)$ & \\
3 & $34(85)$ & $55(85)$ & \\
4 & $1(2)$ & $1(1)$ & \\
otal dose of & 2775 & 3225 & 0.13 \\
dministered FSH (IU) & $(440-7157)$ & $(1000-6300)$ & \\
Basal FSH mUI/ml & $6.6(4.2-12.3)$ & $7.4(2.1-18.3)$ & 0.31 \\
\hline
\end{tabular}

Legend: Continuous variables are presented as means \pm standard deviations (SD), or as medians with their range, in case of skewed distributions. Data are presented as absolute numbers (\%) for ordinal variables. BMI body-mass index; FSH = ; IU international units; IVF in-vitro fertilization; $r$-hCG recombinant human chorionic gonadotropin.

(Figure 1). This difference remained statistically significant after correction for age and indications for IVF in multivariable analysis $(\mathrm{p}=0.032)$. D-dimer levels above the
Table 2 Outcomes of the IVF procedure

\begin{tabular}{lc}
\hline GnRH antagonist protocol, n (\%) & $27 / 105(26)$ \\
Retrieved oocytes, n (range) & $7(2-25)$ \\
Metaphase Il oocytes, n (range) & $5(1-18)$ \\
Biochemical pregnancy only, n (\%) & $5 / 105(5)$ \\
Implantation rate, \% (range) & $19 \%(0$ to 100\%) \\
Live birth rate, n (\%) & $32 / 105(30)$ \\
Clinical pregnancy, n (\%) & $40 / 105(38)$ \\
Twin pregnancy, n (\%) & $9 / 40(22)$ \\
Triplets, n (\%) & - \\
Ectopic pregnancy, n (\%) & $1 / 40(2)$ \\
Pregnancy loss, n (\%) & $7 / 40(17)$ \\
\hline
\end{tabular}

Legend: GnRH Gonadotropin Releasing Hormone.

assay cut-off of positivity $(200 \mathrm{ng} / \mathrm{mL})$ were also associated with a worse outcome $(\mathrm{p}=0.042)$.

One week after the administration of r-hCG, D-dimer values were significantly increased compared to the levels before r-hCG with no significant differences between women with and without a clinical pregnancy $(748 \mathrm{ng} / \mathrm{mL}$ [range 161 to $1057 \mathrm{ng} / \mathrm{mL}$ ] vs. $767 \mathrm{ng} / \mathrm{mL}$ [range 52 to $3140 \mathrm{ng} / \mathrm{mL}], \mathrm{p}=0.88$ ).

\section{Discussion}

In the current study we found that D-dimer levels measured before the administration of $\mathrm{r}$-hCG were predictive of an unfavourable pregnancy outcome.

Relatively few studies with a low number of participants and with several methodological drawbacks explored the changes in haemostatic parameters during IVF [7-10]. In a small case-control study, Rogolino and colleagues found significantly higher concentrations of D-dimer, tissue factor, and protrombin fragments $1+2$ in women hospitalised for OHSS relative to healthy

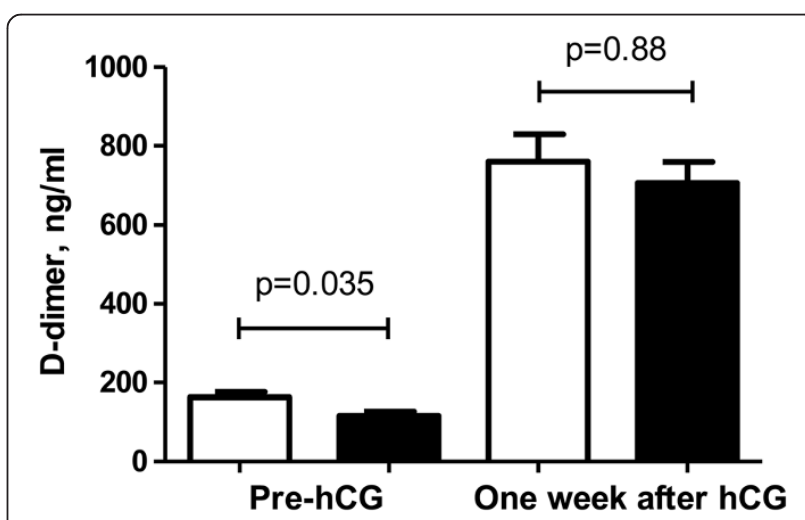

Figure $1 \mathrm{D}$-dimer levels in women with and without a clinical pregnancy. White bars: D-dimer in women without clinical pregnancy; grey bars: D-dimer in women with clinical pregnancy. $r-h C G=$ Recombinant human chorionic gonadotropin. 
controls [7]. Interestingly, the authors reported no differences between controls and women undergoing IVF without OHSS. In another series of 33 women, Curvers and colleagues found only modest changes in the plasma levels of natural anticoagulant proteins despite the considerable variation in hormonal concentrations during the IVF cycle [10].

The D-dimer test is a widely available test which is routinely used in the diagnostic work-up of venous thromboembolic events [11]. In patients at high risk of thrombotic complications, D-dimer measurement helps stratifying the risk of first or subsequent thrombosis and has the potential to identify subgroups warranting primary prophylaxis or prolonged anticoagulation $[12,13]$. To our knowledge, this is the first study to show a negative prognostic value of D-dimer in women undergoing IVF. These results extend previous observations which suggested an association between an unfavourable pregnancy outcome and D-dimer levels in women undergoing IVF and developing OHSS [7]. The present findings need to be considered cautiously due to the size of the included population which did not allow to explore the relationship of Ddimer with live birth rate or identify the D-dimer cut-off that best predict pregnancy success or failure. Future larger studies are warranted to clarify these issues.

D-dimer levels increased considerably one week after the trigger $\mathrm{r}$-hCG injection, in agreement with earlier observations by Biron and colleagues who reported a significant activation of various haemostatic parameters following r-hCG [14]. While the mechanisms behind the activation of blood coagulation following $\mathrm{r}$-hCG remain unclear, the changes in D-dimer concentrations observed in the current study were apparently not related to the final pregnancy outcome. Measurement of D-dimer at later time points after $\mathrm{r}$-hCG could clarify whether these increased D-dimer levels persist long after the r-hCG stimulation or return to pre-stimulation values after a plateau phase. In this latter case, the high concentrations of D-dimer would reflect a transitory peak in blood coagulation activation not sufficiently prolonged to cause thrombosis of the maternal vessels.

In conclusion, we found that D-dimer concentrations seem to predict a higher risk of pregnancy failure in women undergoing IVF. If confirmed in future prospective studies, D-dimer could help identifying a group of patients who could benefit from prophylaxis to increase the pregnancy success rate.

\section{Competing interests}

The authors declare that they have no competing interests.

\section{Authors' contribution}

Study concept and design: EP, GMT; Acquisition of data: VDD; Analysis and interpretation of data: MDN, EP, GMT; Drafting of the manuscript: MDN, GMT; Critical revision of the manuscript for important intellectual content: All authors. Statistical analysis: MDN; Study supervision: GMT. All authors read and approved the final manuscript.

\section{Author details}

'Department of Medical, Oral and Biotechnological Sciences, University "G. D'Annunzio" of Chieti-Pescara, Chieti, Italy. ${ }^{2}$ Department of Vascular Medicine, Academic Medical Center, Amsterdam, Netherlands. ${ }^{3}$ Department of

Medicine and Aging, University "G. D'Annunzio" of Chieti-Pescara, Chieti, Italy.

Received: 14 February 2014 Accepted: 9 May 2014

Published: 22 May 2014

\section{References}

1. Society for Assisted Reproductive Technology and the American Society for Reproductive Medicine: Assisted reproductive technology in the United States: 2000 results generated from the American Society for Reproductive Medicine/Society for Assisted Reproductive Technology Registry. Fertil Steril 2004, 81:1207-1220.

2. Rey E, Kahn SR, David M, Shrier I: Thrombophilic disorders and fetal loss: a meta-analysis. Lancet 2003, 361:901-908.

3. Di Nisio M, Rutjes AWS, Ferrante N, Tiboni GM, Cuccurullo F, Porreca E: Thrombophilia and outcomes of assisted reproduction technologies: a systematic review and meta-analysis. Blood 2011, 118:2670-2678.

4. Middeldorp S, Meijers JCM, van den End AE, van Enk A, Bouma BN, Tans G, Rosing J, Prins MH, Buller HR: Effects on coagulation of levonorgestreland desogestrel containing low dose oral contraceptives: a cross-over study. Thromb Haemost 2000, 84:4-8.

5. Meijers JCM, Middeldorp S, Tekelenburg W, van den Ende AE, Tans G, Prins $\mathrm{MH}$, Rosing J, Buller HR, Bouma BN: Increased fibrinolytic activity during use of oral contraceptives is counteracted by an enhanced Factor $\mathrm{XI}$-independent down regulation of fibrinolysis: a randomized cross-over study of two low-dose oral contraceptives. Thromb Haemost 2000, 84:9-14.

6. Nelson SM: Prophylaxis of VTE in women during assisted reproductive techniques. Thromb Res 2009, 123(Suppl. 3):S8-S15.

7. Rogolino A, Coccia ME, Fedi S, Gori AM, Cellai AP, Scarselli GF, Prisco D, Abbate R: Hypercoagulability, high tissue factor and low tissue factor pathway inhibitor levels in severe ovarian hyperstimulation syndrome: possible association with clinical outcome. Blood Coagul Fibrinolysis 2003, 14:277-282

8. Lox C, Cafiez M, DeLeon F, Dorsett J, Prien S: Hyperestrogenism induced by menotropins alone or in conjunction with leuprolide acetate in in-vitro fertilization cycles: the impact on hemostasis. Fertil Steril 1995, 63:566-570

9. Aune B, Hole KE, Oian P, Hoist N, Osterud B: Does ovarian stimulation for in-vitro fertilization induce a hypercoagulable state? Hum Reprod 1991, 7:925-927.

10. Curvers J, Tans G, Nap AW, Rosing J: Effect of in vitro fertilization treatment and subsequent pregnancy on the protein C pathway. $\mathrm{Br} J$ Haematol 2001, 115:400-407.

11. Di Nisio M, Squizzato A, Rutjes AWS, Buller HR, Zwinderman AH, Bossuyt PMM: Diagnostic accuracy of D-dimer test for exclusion of venous thromboembolism: a systematic review. J Thromb Haemost 2007, 5:296-304.

12. Cosmi B, Legnani C, Cini M, Guazzaloca G, Palareti G: D-dimer and residual vein obstruction as risk factors for recurrence during and after anticoagulation withdrawal in patients with a first episode of provoked deep-vein thrombosis. Thromb Haemost 2011, 105:837-845.

13. Ay C, Dunkler D, Quehenberger P, Marosi C, Zielinski C, Chiriac A-L, Pabinger I: Prediction of venous thromboembolism in cancer patients. Blood 2010, 116:5377-5382.

14. Biron C, Galtier-Dereure I, Rabesandratana H, Bernard I, Aguilar-Martinez P, Schved J-F, Hedon B: Hemostasis parameters during ovarian stimulation for in vitro fertilization: results of a prospective study. Fertil Steril 1997, 67:104-109.

doi:10.1186/1757-2215-7-58

Cite this article as: Di Nisio et al:: Plasma concentrations of D-dimer and outcome of in vitro fertilization. Journal of Ovarian Research 2014 7:58. 